\title{
Knee Osteoarthritis in Type 2 Diabetes Mellitus: Does Insulin Therapy Retard Osteophyte Formation?
}

\author{
Khaled Al-Jarallah ${ }^{a}$ Diaa Shehab ${ }^{a}$ Nabila Abdella ${ }^{a}$ Hisham Al Mohamedy ${ }^{b}$ \\ Mini Abraham ${ }^{a}$ \\ a Department of Medicine, Faculty of Medicine, Kuwait University, and ${ }^{b}$ Department of Medicine, Mubarak \\ Al-Kabeer Hospital, Jabriya, Kuwait
}

\section{Key Words}

Osteoarthritis · Diabetes mellitus · Insulin · Kuwait

\begin{abstract}
Objective: The aim of this study was to investigate whether or not radiographic changes observed in knee osteoarthritis (OA) in type 2 diabetes mellitus (T2DM) patients on insulin therapy differed from those not on insulin. Material and Methods: A cross-sectional study was performed in 311 subjects: 211 T2DM patients and 100 without diabetes (controls) in Mubarak Hospital, Kuwait. Patients were categorized into 3 groups: T2DM patients not on insulin ( $G 1, n=$ 99), T2DM patients on insulin $(G 2, n=112)$ and a nondiabetic control group ( $G 3, n=100)$. Plain X-ray of both knees was used to assess the changes of knee OA and graded using the Kellegren-Lawrence scale (0-4) and the Osteoarthritis Research Society International Atlas grading scale (0-3). A total of 622 knee X-rays were evaluated. SPSS version 21.0 was used for data analysis. Results: A highly significant association ( $p<0.0001$ ) was observed for joint space narrowing (JSN) as well as for osteophyte formation between the three groups. Comparing G2 and G3, a highly significant association ( $p<0.0001$ ) was retained for JSN [201 (89.7\%) vs. 199 (99.5\%)] and for osteophyte formation [26 (11.7\%) vs. 72 (36.0\%)]. Comparing G1 and G2, significantly less osteo-
\end{abstract}

\begin{tabular}{ll}
\hline KARGER & $\begin{array}{l}\text { ( } 2015 \text { S. Karger AG, Basel } \\
1011-7571 / 15 / 0251-0012 \$ 39.50 / 0 \quad \text { Karger }\end{array}$ \\
E-Mail karger@karger.com & $\begin{array}{l}\text { This is an Open Access article licensed under the terms of the } \\
\text { www.karger.com/mpp }\end{array}$ \\
& $\begin{array}{l}\text { Creative Commons Attribution-NonCommercial 3.0 Un- } \\
\text { ported license (CC BY-NC) (www.karger.com/OA-license), } \\
\text { applicable to the online version of the article only. Distribu- } \\
\text { tion permitted for non-commercial purposes only. }\end{array}$
\end{tabular}

phyte formation was noted in $\mathrm{G} 2$ patients compared to $\mathrm{G} 1$ patients [26 (11.7\%) vs. 39 (19.7\%), $p=0.02$ ]. Multivariate logistic regression analysis showed that the G2 group had less chance of osteophyte formation than either the $\mathrm{G} 1$ group or $\mathrm{G} 3$ control group $(\mathrm{OR}=0.294, \mathrm{p}=0.008$ and $\mathrm{OR}=$ $0.098, p<0.001$, respectively). Conclusion: Our findings show that T2DM patients with OA knees on insulin therapy have less radiographic osteophytes compared to T2DM patients not on insulin.

(C) 2015 S. Karger AG, Basel

\section{Introduction}

Osteoarthritis (OA) is one of the most common disabling diseases and has become a growing health care problem because of its increasing prevalence worldwide $[1,2]$. The main phenotypes described are age-related, posttraumatic, hormonal, genetic and metabolic OA. A higher prevalence of $\mathrm{OA}$ has been reported among patients with metabolic syndrome, and diabetes mellitus (DM) was reported as an independent predictor for OA [1-5]. Hyperglycemia and OA interact at both local and systemic levels. Local effects of oxidative stress and advanced glycation end products lead to matrix stiffness, subchondral bone destruction and chondrocyte dysfunc-

Prof. Khaled Al-Jarallah

Department of Medicine, Faculty of Medicine, Kuwait University PO Box 24923

Safat 13110 (Kuwait)

E-Mail aljarallah@ @sc.edu.kw 
tion [6]. As a result of hyperglycemia, low-grade systemic inflammation contributes to a toxic internal environment that exacerbates OA $[1,6]$. The other suggested reason attributed to the correlation between DM and OA is diabetic peripheral neuropathy leading to muscle weakness and joint laxity in OA [1]. The lack of agreement between X-ray evidence of OA and a patient's report of pain at the site has also been attributed to diabetic neuropathy [7]. Still, because of the inconsistencies and limitations of observational studies, a conclusive association between $\mathrm{DM}$ and $\mathrm{OA}$ has not been determined $[1,8]$. The incidence of an aging population, metabolic syndrome and $\mathrm{OA}$ is exponentially increasing worldwide [1-4], and this trend is also seen in the Kuwaiti population $[9,10]$. Hence, research in this field for new treatments of OA has become mandatory.

Concepts of insulin therapy for OA have been proposed in the past decades [11]. The anabolic effect of insulin on connective tissue and its role as a potent growth factor for connective tissue has been described [11-13]. Early studies performed on whole animals, organs or tissues indicated that insulin is required for the optimal production of sulfated mucopolysaccharides and collagen, two major components of connective tissue [14-17]. In experiments conducted on articular cartilage explants harvested from patients undergoing knee joint replacement, Cai et al. [11] reported that insulin inhibited nitric oxide production and stimulated matrix synthesis by counteracting the deleterious effect of interleukin 1 (IL1), high levels of which are usually found in arthritic joints. Another observation relating to insulin therapy was made by Nieves-Plaza et al. [8], who reported that patients with DM who did not use insulin for their condition had a 4.44-fold increased risk of hand or knee OA.

Radiographic features of bone and cartilage seen in $\mathrm{OA}$ are the result of degenerative changes as evidenced by articular cartilage thinning and joint space narrowing (JSN), and proliferative changes evidenced by concomitant repair activity with new cartilage and bone growth resulting in osteophytosis and subchondral sclerosis. Limited studies $[8,12]$ have been conducted on the effect of insulin on radiographic features and osteophyte formation in OA in diabetic patients. Horn et al. [12] reported that osteophyte formation, as well as other radiographic indicators of joint repair, are less marked in type $2 \mathrm{DM}$ (T2DM) patients with OA compared to nondiabetic patients with OA. Hence, the aim of our study was to evaluate whether or not the radiographic changes of knee OA differed between T2DM patients on insulin and those not on insulin therapy.

\section{Subjects and Methods}

\section{Study Population}

A cross-sectional study was performed on a total of 311 subjects consisting of 211 T2DM patients and 100 nondiabetic control subjects during a period of 12 months, from September 2012 to August 2013. Consecutive T2DM patients with knee pain were enrolled from the diabetic clinics of Mubarak Al-Kabeer Hospital, Kuwait. T2DM was defined as a fasting plasma glucose level $\geq 7.0 \mathrm{mmol} / \mathrm{l}$ on at least two occasions, or $\mathrm{Hb} \mathrm{A} 1 \mathrm{C} \geq 6.5 \%$ [18].

Among the 211 T2DM patients ( 86 males, 125 females; aged $38-86$ years), 20 were only on an exercise regime and not on any medication for diabetes, and 79 were on oral hypoglycemic agents alone. These 99 patients were grouped as the noninsulin group (G1). Thirty-six patients were on insulin alone and 76 were on both insulin and oral hypoglycemic agents. These 112 patients were included in the category of diabetic patients on insulin (G2). The duration of insulin therapy was $\geq 5$ years, with a total insulin daily requirement ranging from 40 to $80 \mathrm{IU}$, given subcutaneously in 1-3 divided doses. Nondiabetic subjects referred for knee pain to Physical Medicine and Rehabilitation clinics served as controls (G3). Exclusion criteria were patients with inflammatory rheumatic diseases, malignancy, hyperparathyroidism, renal disease or liver disease. All the patients were studied according to the protocol approved by the Ethics Committees of the Faculty of Medicine, Kuwait University, and the Ministry of Health in accordance with the provisions for human research established in the Declaration of Helsinki.

Written informed consent was obtained from each participant. Details of knee pain, clinical examinations of the knees, height, weight, BMI (calculated using the standard formula: weight divided by height in meters squared), diabetic history with regard to the duration of diabetes and diabetic treatment in subjects with T2DM were collected and recorded on Performa. Biochemical parameters were measured as part of their routine diabetic workup.

\section{Study Methodology}

Knee function was assessed using the Lequesne index for knee OA [19], which is a ten-question survey given to patients suffering from the condition. It is composed of five questions relating to pain or discomfort, one question dealing with maximum distance walked and four questions about activities of daily living. The total questionnaire is scored on a scale from 0 to 24 . A Lequesene index up to 10 is considered to be good and $>10$ is viewed as poor, with higher scores indicating that there is greater functional impairment.

A standard X-ray weight-bearing, anterior-posterior image of both knees was taken for all patients. OA of the knee was ascertained using the American College of Rheumatology classification criteria $[20,21]$. Both the Kellegren-Lawrence (K-L) the Osteoarthritis Research Society International (OARSI) classification systems were used to assess the radiographic images. The K-L grades (range 0-4) are as follows: 0 - normal; 1 - doubtful narrowing of joint space and possible osteophyte lipping; 2 - definite osteophytes and possible narrowing of joint space; 3 - definite narrowing of joint space and some sclerosis and possible deformity of bone ends; 4 - significant osteophytes, marked narrowing of joint space, severe sclerosis and definite deformity of bone ends [22]. The OARSI classification grades (range $0-3$ ) are as follows: 0 - normal; 1 - mild narrowing (1-33\% narrowed); 2 - moderate narrowing (34-66\%); 3 - severe narrowing (67-100\%). The OARSI grades were given for medial and lateral tibiofemoral compartments. [23]. 
Statistical Analysis

Results were analyzed using the Statistical Package for Social Sciences version 21.0 (SPSS Inc., Chicago, Ill., USA). Descriptive statistics were used to describe the variables. Categorical variables were compared by $\chi^{2}$ test and mean values of continuous variables were compared by ANOVA. Binary logistic regression analysis was used to ascertain the association of osteophyte formation with insulin therapy with and without adjustment for the confounding effects of gender, BMI and age. Values of $\mathrm{p}<0.05$ were considered to be statistically significant.

\section{Results}

A total of 622 knee X-rays were evaluated. Two investigators (K.J. and D.S.) assessed and scored all the radiographs, blinded to the clinical details of subjects. The inter-reader agreement was $90 \%$ for cases of JSN and $81 \%$ for osteophytes cases. Differences were resolved by the readers reaching a consensus interpretation of radiographs.

The demographic characteristics of the participants in the cohort are shown in table 1. Among the cohorts the differences between gender and BMI were statistically significant ( $\mathrm{p}<0.001$ and $<0.039$, respectively). The evaluation of functional or pain scores (Lequesne index score) among cohorts did not show any statistically significant differences $(\mathrm{p}=0.106)$.

Blood sugar was controlled with a target of $\mathrm{HbA1C}$ between 7 and $8 \%$ in our diabetic subjects. The mean $\mathrm{HbA} 1 \mathrm{C} \%$ value for the nondiabetic control group (G3) was $5.5 \pm 2.0$, and there was no statistical difference in values of $\mathrm{HbA1C}$ between the noninsulin (G1) and insulin (G2) T2DM patients (7.6 \pm 2.0 vs. $7.9 \pm 2.1$, respectively, $\mathrm{p}=0.124)$.

The mean \pm SD K-L score was similar in the three groups: $2.22 \pm 0.7$ (G1), $2.27 \pm 0.8(\mathrm{G} 2)$ and $2.37 \pm 0.8$ (G3; $\mathrm{p}>0.161)$. However, a significant difference $(\mathrm{p}=0.016)$ was observed in the grading of K-L scores among cohorts, with a higher grading (i.e. grade 4 ) observed in the nondiabetic control group [G3: $16(8.0 \%)$ ] than in the diabetic patients [G1: $8(4.0 \%)$ and G2: $8(3.6 \%)]$.

OARSI grading showed highly significant differences ( $p>0.0001$ ) between G1, G2 and G3, respectively, for JSN [175 (88.4\%) vs. $201(89.7 \%)$ vs. $199(99.5 \%)]$ and osteophyte formation [39(19.7\%) vs. $26(11.7 \%)$ vs. $72(36.0 \%)]$. Comparing the insulin group and control subjects (G2 vs. G3), the highly significant difference $(\mathrm{p}<0.0001)$ for both JSN [201 (89.7\%) vs. 199 (99.5\%)] and for osteophyte formation [26 (11.7\%) vs. $72(36.0 \%)]$ was maintained, whereas comparing the insulin and noninsulin T2DM
Table 1. Demographic characteristics of the T2DM patients and controls

\begin{tabular}{|c|c|c|c|c|}
\hline & $\begin{array}{l}\text { G1: non- } \\
\text { insulin } \\
(n=99)\end{array}$ & $\begin{array}{l}\text { G2: insulin } \\
(\mathrm{n}=112)\end{array}$ & $\begin{array}{l}\text { G3: controls } \\
(\mathrm{n}=100)\end{array}$ & $\mathrm{p}$ \\
\hline \multicolumn{5}{|l|}{ Mean age $\pm \mathrm{SD}$} \\
\hline $\begin{array}{l}\text { years } \\
\text { Range }\end{array}$ & $\begin{array}{l}57.8 \pm 8.9 \\
39-80\end{array}$ & $\begin{array}{l}60.3 \pm 9.5 \\
38-79\end{array}$ & $\begin{array}{l}57.1 \pm 9.4 \\
27-80\end{array}$ & 0.059 \\
\hline$<50$ years & $22(23.2)$ & $15(13.6)$ & $20(20.0)$ & 0.189 \\
\hline$>50$ years & $72(76.6)$ & $95(86.4)$ & $80(80.0)$ & \\
\hline \multicolumn{5}{|l|}{ Gender } \\
\hline Male & $46(46.5)$ & $40(35.7)$ & $12(12.0)$ & $<0.001$ \\
\hline Female & $53(53.5)$ & $72(64.3)$ & $88(88.0)$ & \\
\hline \multicolumn{5}{|c|}{ Duration of diabetes } \\
\hline$<10$ years & $35(38.5)$ & $17(15.7)$ & - & $<0.001$ \\
\hline$>10$ years & $56(61.5)$ & $91(84.3)$ & - & \\
\hline \multicolumn{5}{|l|}{ BMI } \\
\hline$<30$ & $34(47.9)$ & $31(35.6)$ & $45(54.9)$ & 0.039 \\
\hline$>30$ & $37(52.1)$ & $56(64.4)$ & $37(45.1)$ & \\
\hline \multicolumn{5}{|c|}{ Functional score (Lequesne index score) } \\
\hline Good $(\leq 10)$ & $44(55.7)$ & $36(40.4)$ & $42(42.9)$ & 0.106 \\
\hline Poor $(>10)$ & $35(44.3)$ & $53(59.6)$ & $56(57.1)$ & \\
\hline
\end{tabular}

Values are n (\%) unless otherwise indicated.

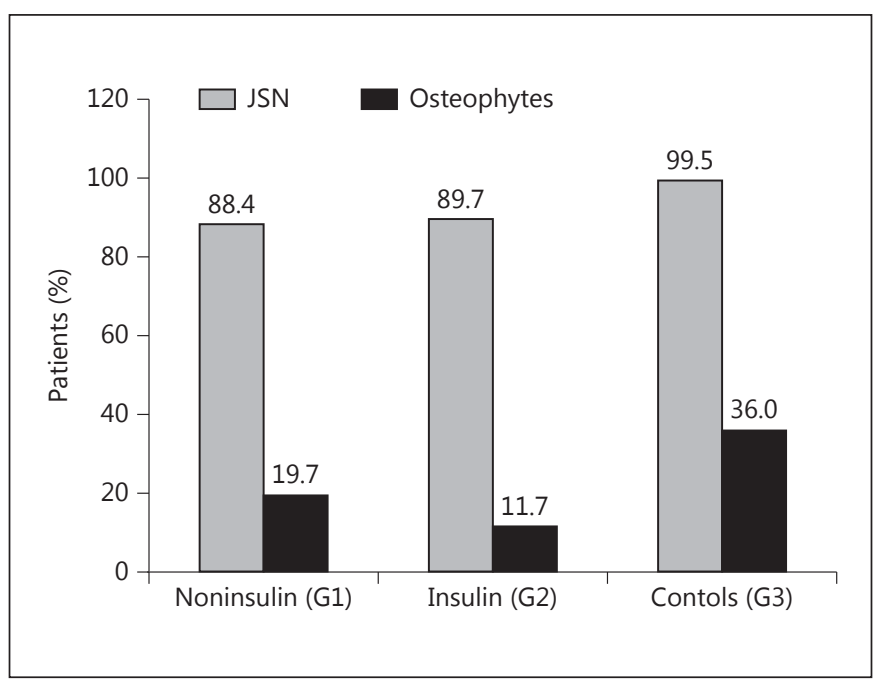

Fig. 1. The percentage of study subjects with JSN and osteophytes.

patients (G1 vs. G2) revealed no association [175 (88.4\%) vs. 201 ( $89.7 \%), \mathrm{p}=0.386$ ] for JSN, although significantly less osteophytes were observed in G2 [26 (11.7\%)] compared to G1 patients [39 (19.7\%), p $=0.022$; table 2]. The percentage of G1, G2 and G3 subjects with JSN and osteophytes are shown in figure 1 .
14

Med Princ Pract 2016;25:12-17 DOI: $10.1159 / 000441418$
Al-Jarallah/Shehab/Abdella/

Al Mohamedy/Abraham 
Table 2. Association between radiographic K-L score, and OARSI score for JSN and osteophyte formation between the noninsulin, insulin and control groups

\begin{tabular}{|c|c|c|c|c|c|}
\hline & $\begin{array}{l}\text { Total } \\
(\mathrm{n}=622)\end{array}$ & $\begin{array}{l}\text { G1: noninsulin } \\
(\mathrm{n}=198)\end{array}$ & $\begin{array}{l}\text { G2: insulin } \\
(\mathrm{n}=224)\end{array}$ & $\begin{array}{l}\text { G3: controls } \\
(n=200)\end{array}$ & $\mathrm{p}$ \\
\hline \multicolumn{6}{|l|}{ Radiographic K-L score } \\
\hline Mean score \pm SD (range) & & $2.22 \pm 0.7(0-4)$ & $2.27 \pm 0.8(0-4)$ & $2.37 \pm 0.8(1-4)$ & 0.161 \\
\hline Grade 0 & $12(1.9)$ & $4(2.0)$ & $8(3.6)$ & 0 & 0.016 \\
\hline Grade 1 & $72(11.6)$ & $23(11.6)$ & $20(8.9)$ & $29(14.5)$ & \\
\hline Grade 2 & $296(47.6)$ & $105(53.0)$ & $107(47.8)$ & $84(42.0)$ & \\
\hline Grade 3 & $210(33.8)$ & $58(29.3)$ & $81(36.2)$ & $71(35.5)$ & \\
\hline Grade 4 & $32(5.1)$ & $8(4.0)$ & $8(3.6)$ & $16(8.0)$ & \\
\hline \multicolumn{6}{|l|}{$J S N$} \\
\hline Absent & $47(7.6)$ & $23(11.6)$ & $23(10.3)$ & $1(0.5)$ & $<0.0001$ \\
\hline Present & $572(92.4)$ & $175(88.4)$ & $201(89.7)$ & $199(99.5)$ & \\
\hline \multicolumn{6}{|l|}{ Osteophyte formation } \\
\hline Absent & $485(78.0)$ & $159(80.3)$ & $198(88.4)$ & $128(64.0)$ & $<0.0001$ \\
\hline Present & $137(22.0)$ & $39(19.7)$ & $26(11.7)$ & $72(36.0)$ & \\
\hline Grade 0 & $483(77.5)$ & $158(79.8)$ & $198(88.4)$ & $127(63.5)$ & $<0.0001$ \\
\hline Grade 1 & $114(18.3)$ & $28(14.1)$ & $15(6.7)$ & $71(35.5)$ & \\
\hline Grade 2 & $20(3.2)$ & $10(5.1)$ & $8(3.6)$ & $2(1.0)$ & \\
\hline Grade 3 & $5(0.8)$ & $2(1.0)$ & $3(1.3)$ & 0 & \\
\hline
\end{tabular}

Values are $\mathrm{n}(\%)$ unless otherwise indicated. Association for JSN between G2 and G3: $\mathrm{p}<0.0001$, and between G1 and G2: $p>0.05$. Association for osteophyte formation between G2 and G3: $p<0.001$, and between G1 and $\mathrm{G} 2: \mathrm{p}=0.022$.

Binary logistic regression analysis for osteophyte formation between the cohorts generated odds ratio (OR), $95 \%$ confidence interval (CI) and $\mathrm{p}$ values for the predictive role of various variables, as shown in table 3 . Unadjusted analysis for osteophyte formation between G2 and $\mathrm{G} 3$ showed a significant association $(\mathrm{OR}=0.233,95 \%$ $\mathrm{CI}=0.142-0.385, \mathrm{p}<0.001$ ), which persisted after adjusting for gender, $\mathrm{BMI}$ and age $(\mathrm{OR}=0.098,95 \% \mathrm{CI}=0.042$ $0.230, \mathrm{p}<0.001$ ). Similarly, between G1 and G2 there was also a significant association for unadjusted analysis $(\mathrm{OR}=0.535,95 \% \mathrm{CI}=0.312-0.917, \mathrm{p}<0.023)$ which remained after adjustment for gender, $\mathrm{BMI}$ and age $(\mathrm{OR}=$ $0.294,95 \% \mathrm{CI}=0.119-0.722, \mathrm{p}<0.008)$.

\section{Discussion}

In this study the degree of osteophyte formation as well as cartilage loss was greater in control subjects and in T2DM patients not receiving insulin than T2DM patients on insulin therapy. The lack of a difference in the K-L scores relative to other parameters that revealed a differ-
Table 3. Binary logistic regression analysis for osteophyte formation

\begin{tabular}{lccc}
\hline Variable & OR & $95 \%$ CI & p value \\
\hline T2DM patients on insulin and controls (G2 and G3) & \\
Crude OR for each variable (unadjusted) & & \\
G2 & 0.233 & $0.142-0.385$ & $<0.001$ \\
G3 (reference) & 1.000 & & \\
Multivariate analysis (adjusted for all variables) & \\
G2 & 0.098 & $0.042-0.230$ & $<0.001$ \\
G3 (reference) & 1.000 & & \\
\hline
\end{tabular}

T2DM patients not on insulin and on insulin (G1 and G2)

Crude OR for each variable (unadjusted)

$\begin{array}{lrll}\text { G2 } & 0.535 & 0.312-0.917 & 0.023 \\ \text { G1 (reference) } & 1.000 & & \\ \text { ultivariate analysis (adjusted for all variables) } & \\ \text { G2 } & 0.294 & 0.119-0.722 & 0.008 \\ \text { G1 (reference) } & 1.000 & & \end{array}$

The dependent variable was osteophytes $( \pm)$ and the independent variable was patient group. 
ence between the patient groups could be explained as follows. Whilst both K-L and OARSI are semiquantitative radiographic tools that provide an assessment of the extent of knee OA, the K-L scoring system is used to assess the OA status of the entire tibiofemoral joint, whereas the OARSI scoring system describes the extent of OA in the medial and lateral compartments separately. Therefore, they are not designed to be interchangeable.

Our findings are consistent with those of Cai et al. [11] who reported a beneficial effect of insulin in the treatment of OA. OA is characterized by loss of cartilage matrix molecules, proteoglycans (PG) and collagens. Articular cartilage from diabetic mice had lower basal levels of PG synthesis than nondiabetic controls. Cai et al. [11] demonstrated that local insulin treatment increased PG synthesis and thus increased matrix synthesis as well as decreasing matrix breakdown. Matrix breakdown is one of the earliest and most destructive features of arthritis. Osteophyte formation and cartilage loss, the most typical pathologic findings in OA patients, is secondary to matrix loss and breakdown. Thus, inhibition of this process and stimulation of new matrix molecules would be expected to promote tissue and joint repair.

Another mechanism whereby insulin decreases matrix breakdown is through the inhibition of aggrecanase activity. In addition, insulin inhibits nitric oxide production and antagonizes IL-1, which each have detrimental effects on chondrocytes and other cell types within the joint. Furthermore, insulin is a poor mitogen of osteoblasts and fibroblasts, which are necessary for the formation of bony nodules or osteophytes. Cai et al. [11] also showed that the response of cartilage to insulin did not diminish with age or disease; thus, insulin has potential as a treatment for OA at different stages of joint destruction.

It is not clear how systemic or exogenous inulin administration may differ in its impact on the skeleton compared with local delivery, which would have a much lesser impact on glycemic response and metabolism [13]. Spanheimer [24] reported that systemic administration of insulin can at least partially reverse the defects in connective tissue metabolism. While insulin may have direct effects on connective tissue, reversal of defects in connective tissue metabolism by the systemic administration of insulin could be due to circulating factor(s) induced by insulin and not due to the direct effect of insulin on connective tissue.

Our findings were consistent with those of Horn et al. [12] who observed that osteophytes were less marked in T2DM patients than in nondiabetic patients with OA. A possible explanation for these observations and the effect of insulin therapy on osteophytes formation could be related to the fact that diabetic microvascular disease attenuates osteogenesis and diminished proteoglycan synthesis leading to inhibition of insulin-dependent mesenchymal cell proliferation, which may attribute to reduced osteophyte formation in diabetic patients. Another postulation for osteophyte formation is changes in the stress distribution in periarticular and subchondral bone. Diminished physical activity in DM patients was attributed to the fewer osteophytes observed in DM patients in the study [12].

In our study, only $45 \%$ of the nondiabetic controls were obese as compared to $52.1 \%$ of the noninsulin and $64.4 \%$ of the insulin-treated T2DM patients $(\mathrm{p}=0.039)$. Alterations in connective tissue metabolism in the OA joint due to DM may have been compounded by the metabolic effects of obesity. However, after adjustment for BMI (as well as age and gender), our T2DM patients on insulin treatment remained less predictive for the presence of radiographic osteophytes.

Obesity in itself is a risk factor for OA independent of $\mathrm{DM}$ and is associated with insulin resistance and hyperinsulinemia $[25,26]$. In animal experiments, Griffin et al. [27] demonstrated that diet-induced obesity increases the risk of symptomatic features of OA through changes in musculoskeletal function and pain-related behaviors. Furthermore, the independent association of systemic adipokine levels with knee OA severity supports a role for adipose-associated inflammation in the molecular pathogenesis of obesity-induced OA. Issa and Griffin [28] reviewed the pathobiology of obesity and OA and highlighted the role of proinflammatory metabolic factors in increasing the risk of OA. Adipose tissue, in particular infrapatellar fat, is a local source of inflammatory mediators that are increased with obesity and have been shown to increase cartilage degradation in cell and tissue culture models. Furthermore, Altuntaş et al. [29] investigated the association between articular damage in diabetes at gene expression and protein levels, and concluded that insulin reduces the amount of ADAMTS12 (A disintegrin and metalloproteinase with thrombospondin motifs 12), an enzyme in humans that is encoded by the ADAMTS12 gene. This enzyme causes a delayed recovery of cartilage tissue and its reduction by insulin can contribute to the recovery of cartilage tissue. These experiments were conducted on OUMS-27 cell lines from chondrosarcoma cells which have chondrocytic properties.

Our study design was cross-sectional and assessed only anteroposterior knee films, which are routinely used
16

Med Princ Pract 2016;25:12-17 DOI: $10.1159 / 000441418$
Al-Jarallah/Shehab/Abdella/

Al Mohamedy/Abraham 
in population surveys. However, radiographic assessment by two experienced musculoskeletal physicians with an inter-reader agreement of $90 \%$ for JSN and $81 \%$ for osteophytes was good enough to achieve the aims of the study.

\section{Conclusion}

In this study of OA of the knee, T2DM patients on insulin therapy had less radiographic osteophytes than both T2DM patients who were not on insulin and healthy control subjects. As the impact of insulin treatment on the biomechanical properties of the intact skeleton remains unclear, further longitudinal studies are needed to address issues such as the mode and dose of administration to maximize insulin effectiveness for OA management, methods to increase the stability of locally administered insulin, and also to delineate the mechanisms underlying these observations.

\section{Disclosure Statement}

The authors have no conflicts of interest to declare in relation to this work.

\section{References}

1 Berenbaum F: Diabetes-induced osteoarthritis: from a new paradigm to a new phenotype. Ann Rheum Dis 2011;70:1354-1356.

-2 Velasquez MT, Katz JD: Osteoarthritis: another component of metabolic syndrome? Metab Syndr Relat Disord 2010;8:295-305.

-3 Jérémie S, Francis B: Is osteoarthritis a metabolic disease? Joint Bone Spine 2013;80:568573.

4 Yoshimura N, Muraki S, Oka H, et al: Accumulation of metabolic risk factors such as overweight, hypertension, dyslipidaemia, and impaired glucose tolerance raises the risk of occurrence and progression of knee osteoarthritis: a 3-year follow-up of the ROAD study. Osteoarthritis Cartilage 2012;20:1217-1226.

5 Schett G, Kleyer A, Perricone C, et al: Diabetes is an independent predictor for severe osteoarthritis: results from a longitudinal cohort study. Diabetes Care 2013;36:403-409.

6 Zhuo Q, Yang W, Chen J, et al: Metabolic syndrome meets osteoarthritis. Nat Rev Rheumatol 2012;8:729-737.

7 Leaverton PE, Peregoy J, Fahlman L, et al: Does diabetes hide osteoarthritis pain? Med Hypotheses 2012;78:471-474.

8 Nieves-Plaza M, Castro-Santana LE, Font YM, Mayor AM, et al: Association of hand or knee osteoarthritis with diabetes mellitus in a population of Hispanics from Puerto Rico. J Clin Rheumatol 2013;19:1-6.

-9 Nabila A, Moustafa M, Amani D, et al: Pattern of non-insulin dependent diabetes mellitus in Kuwait. Diabetes Res Clin Pract 1995;29:129_ 136.

10 Al-Awadi A, Olusi S, Moussa M, et al: Musculoskeletal pain, disability and health- seeking behavior in adult Kuwaitis using a validated Arabic Version of the WHO-ILAR COPCORD Core Questionnaire. Clin Exp Rheumatol 2004;22:177-183.
11 Cai L, Okumu FW, Cleland JL, et al: A slow release formulation of insulin as a treatment for osteoarthritis. Osteoarthritis Cartilage 2002;10:692-706.

12 Horn CA, Bradley JD, Brandt KD, et al: Impairment of osteophyte formation in hyperglycemic patients with type II diabetes mellitus and knee osteoarthritis. Arthritis Rheum 1992;35:336-342.

13 Thrailkill KM, Lumpkin CK Jr, Bunn RC, et al: Is insulin an anabolic agent in bone? Dissecting the diabetic bone for clues. Am J Physiol Endocrinol Metab 2005;289:E735-E745.

14 Anthony S, Michael S: Stimulation of growth and mucopolysaccharide synthesis by insulin treatment of chick embryo chondrocytes in cell culture. Gen Comp Endocrinol 1975;25: 432-446.

15 Peck WA, Messinger K: Nucleoside and ribonucleic acid metabolism in isolated bone cells effects of insulin and cortisol in vitro. J Biol Chem 1970;245:2722-2729.

16 Canalis EM, Dietrich JW, Maina DM, et al: Hormonal control of bone collagen synthesis in vitro: effects of insulin and glucagon. Endocrinology 1977;100:668-674.

17 Maor G, Silbermann M, von der Mark K, et al: Insulin enhances the growth of cartilage in organ and tissue cultures of mouse neonatal mandibular condyle. Calcif Tissue Int 1993; 52:291-299.

18 American Diabetes Association: Standards of medical care in diabetes - 2013. Diabetes Care 2013;36(suppl 1):S11-S66.

19 Lequesne MG, Mery C, Samson M, et al: Indexes of severity for osteoarthritis of the hip and knee: validation - value in comparison with other assessment tests. Scand J Rheumatol Suppl 1987;65:85-89.
20 Kellgren JH, Lawrence JS (eds): The Epidemiology of Chronic Rheumatism: Atlas of Standard Radiographs of Arthritis. Oxford, Blackwell Scientific, 1963.

21 Spector TD, Cooper C, Cushnaghan J, et al: A Radiographic Atlas of Knee Osteoarthritis. London, Springer, 1992, pp 1-15.

22 Kellgren JH, Lawrence JS: Radiological assessment of osteo-arthritis. Ann Rheum Dis 1957; 16:494-502.

23 Altman RD, Hochberg M, Murphy WA Jr, et al: Atlas of individual radiographic features in osteoarthritis. Osteoarthritis Cartilage 1995; 3(suppl A):3-70.

24 Spanheimer RG: Correlation between decreased collagen production in diabetic animals and in cells exposed to diabetic serum: response to insulin. Matrix 1992;12:101107.

25 Felson DT, Anderson JJ, Naimark A, et al Obesity and knee OA: the Framingham Study. Ann Intern Med 1988;109:18-24.

26 Olefsky JM, Kolterman OG: Mechanisms of insulin resistance in obesity and noninsulindependent (type II) diabetes. Am J Med 1981; 70:151-168.

27 Griffin TM, Fermor B, Huebner JL, et al: Dietinduced obesity differentially regulates behavioral, biomechanical, and molecular risk factors for osteoarthritis in mice. Arthritis Res Ther 2010;12:R130.

28 Issa RI, Griffin TM: Pathobiology of obesity and osteoarthritis: integrating biomechanics and inflammation. Pathobiol Aging Age Relat Dis 2012;2:17470.

29 Altuntaș A, Akyol S, Adam B, et al: ADAMTS12 depletion by insulin in OUMS-27 human chondrosarcoma cells. Arch Rheumatol 2015;30:i-viii. 